\title{
Generation of optimal packings from optimal packings
}

\author{
Thierry Gensane * \\ Laboratoire de Mathématiques Pures et Appliquées Joseph Liouville \\ 50, rue F. Buisson, BP 699, 62228 Calais Cedex, FRANCE \\ gensane@lmpa.univ-littoral.fr
}

Submitted: Oct 2, 2008; Accepted: Feb 13, 2009; Published: Feb 20, 2009

Mathematics Subject Classifications: 52C15, 05B40

\begin{abstract}
We define two notions of generation between the various optimal packings $\mathcal{Q}_{m}^{K}$ of $m$ congruent disks in a subset $K$ of $\mathbb{R}^{2}$. The first one that we call weak generation consists in getting $\mathcal{Q}_{n}^{K}$ by removing $m-n$ disks from $\mathcal{Q}_{m}^{K}$ and by displacing the $n$ remaining congruent disks which grow continuously and do not overlap. During a weak generation of $\mathcal{Q}_{n}^{K}$ from $\mathcal{Q}_{m}^{K}$, we consider the contact graphs $\mathcal{G}(t)$ of the intermediate packings, they represent the contacts disk-disk and disk-boundary. If for each $t$, the contact graph $\mathcal{G}(t)$ is isomorphic to the largest common subgraph of the two contact graphs of $\mathcal{Q}_{n}^{K}$ and $\mathcal{Q}_{m}^{K}$, we say that the generation is strong. We call strong generator in $K$, an optimal packing $\mathcal{Q}_{m}^{K}$ which generates strongly all the optimal $\mathcal{Q}_{k}^{K}$ with $k<m$. We conjecture that if $K$ is compact and convex, there exists an infinite sequence of strong generators in $K$. When $K$ is an equilateral triangle, this conjecture seems to be verified by the sequence of hexagonal packings $\mathcal{Q}_{\Delta(k)}^{K}$ of $\Delta(k)=k(k+1) / 2$ disks. In this domain, we also report that up to $n=34$, the Danzer graph of $\mathcal{Q}_{n}^{K}$ is embedded in the Danzer graph of $\mathcal{Q}_{\Delta(k)}^{K}$ with $\Delta(k-1) \leq n<\Delta(k)$. When $K$ is a circle, the first five strong generators appears to be the hexagonal packings defined by Graham and Lubachevsky. When $K$ is a square, we think that our conjecture is verified by a series of packings proposed by Nurmela and al. In the same domain, we give an alternative conjecture by considering another packing pattern.
\end{abstract}

\section{Introduction}

The search of the densest packing of $n$ non-overlapping equal disks in a compact set of the plane is a classical problem of discrete geometry. An introductory bibliography on this subject can be found in $[1,8]$ and a large collection of packing problems in [2]. The

\footnotetext{
${ }^{*}$ In memory of Daniel Gensane (1934-2008), my father.
} 
literature focusses mainly on packings in a square, a disk or an equilateral triangle. All best known packings up to 300 disks in the square and 500 disks in the circle are given on the website [12].

The aim of this paper is to formulate some conjectures about a link of generation which seems to exist among the various optimal packings in a domain $K$. It has been remarked in [6] that some dense packings are obtained by removing one disk from a given packing and by a a small rearrangement of the disks. We will say that an optimal packing $\mathcal{Q}_{m}^{K}$ of $m$ disks in $K$ is a weak generator of an optimal packing $\mathcal{Q}_{n}^{K}$, if it is possible to obtain $\mathcal{Q}_{n}^{K}$ by removing $m-n$ disks from $\mathcal{Q}_{m}^{K}$ and by displacing the $n$ remaining congruent disks whose size is increasing and which do not overlap. Let us look at the first row of Fig. 1: we find from left to right, the optimal packing $\mathcal{Q}_{9}^{\square}$, an intermediate packing $\mathcal{Q}(t)$ parameterized on $[0,1]$ and the optimal packing $\mathcal{Q}_{8}^{\square}$. After removing the central disk of $\mathcal{Q}_{9}^{\square}$, the eight remaining disks behave like biological cells which search some space in order to increase their size. Moreover, it is remarkable that for each $t \in] 0,1[$, the contact graph of $\mathcal{Q}(t)$ (whose edges represent the contacts disk-disk or disk-boundary) is isomorphic to the largest maximal common subgraph of the contact graphs of $\mathcal{Q}_{9}^{\square}$ and $\mathcal{Q}_{8}^{\square}$. This observation is also verified when $\mathcal{Q}_{9}^{\square}$ generates $\mathcal{Q}_{8}^{\square}$ and $\mathcal{Q}_{7}^{\square}$ in the second and third rows of Fig. 1. When such a transformation exists between two packings, we say that the generation from one to the other is strong. To find the largest maximal common subgraph of two graphs $G_{1}, G_{2}$ is a NP-hard problem [3]. The restriction of this problem to induced common subgraphs leads to the equivalent problem: How to find the largest clique in the graph product $G_{1} \times G_{2}$, see [16]. Unfortunately, the largest common subgraph which

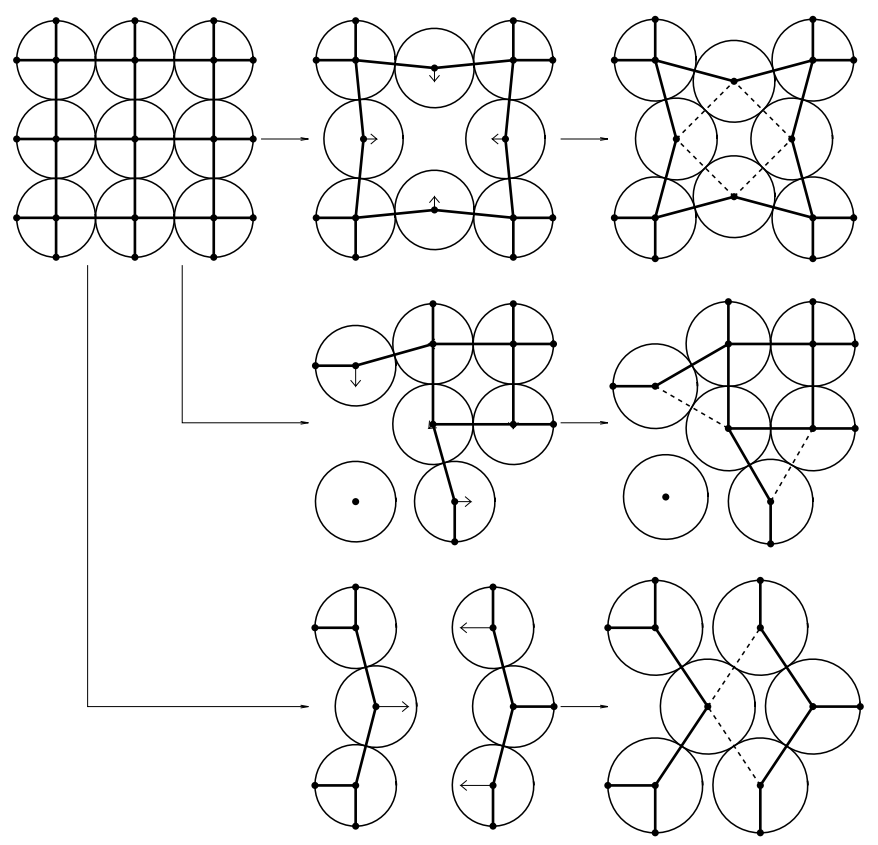

Figure 1: Strong generations of $\mathcal{Q}_{8}^{\square}, \mathcal{Q}_{7}^{\square}, \mathcal{Q}_{6}^{\square}$ from $\mathcal{Q}_{9}^{\square}$. 
appears when a packing generates strongly another one is not induced (neither connected in general). We emphasize that all largest maximal common subgraphs displayed in this paper have been found without the help of a computer and must be considered as the best common subgraphs we found. We differ to a subsequent work the use of codes which compute the largest common subgraphs of two contact graphs. This should be the first step in order to verify or invalidate the conjectures presented in this paper.

Let us now examine in Fig. 2, another series of largest common subgraphs $\mathcal{G}_{k}^{\circ} \cap \mathcal{G}_{19}^{\circ}$ relative to the generation of the optimal packings $\mathcal{Q}_{k}^{\circ}$ of $k$ disks in the circle from the optimal packing $\mathcal{Q}_{19}^{\circ}$. The visualization of the largest common subgraphs allows to imagine how $\mathcal{Q}_{19}^{\circ}$ generates strongly $\mathcal{Q}_{k}^{\circ}$ after removing the disks whose centers are not in the common structure. We can easily convince ourselves that $\mathcal{Q}_{19}^{\circ}$ is a strong (resp. weak) generator in the circle in the sense that it generates strongly (resp. weakly) all of the previous optimal packings $\mathcal{Q}_{k}^{\circ}$ (the packing $\mathcal{Q}_{18}^{\circ}$ is directly obtained by removing one disk of $\mathcal{Q}_{19}^{\circ}$ and the generations of the packings $\mathcal{Q}_{1}^{\circ}, \mathcal{Q}_{2}^{\circ}, \ldots, \mathcal{Q}_{7}^{\circ}$ - found in [12] - are easily settled). A natural question is to know whether there exists an infinity of generators in a domain $K$. The following conjecture can be weakened by changing "strong" for "weak" .

Conjecture 1 Let $K$ be a compact convex subset of $\mathbb{R}^{2}$. There exists an infinite sequence of integers $n_{1}<n_{2}<\ldots<n_{k}<\ldots$ such that each optimal packing $\mathcal{Q}_{n_{k}}^{K}$ of $n_{k}$ disks is a strong generator in $K$.

After Section 2 in which we give our notation and definitions, we precise Conjecture 1 in Section 3 when $K$ is the equilateral triangle. In Section 4 , we deal with the disk packings in the square. We will not come back to Conjecture 1 when $K$ is a circle. Indeed, at the time being we are not able to identify a regular pattern which produces an infinite series of optimal packings in circle. Nevertheless, it is reasonable to think that the five hexagonal packings $\mathcal{Q}_{7}^{\circ}, \mathcal{Q}_{19}^{\circ}, \mathcal{Q}_{37}^{\circ}, \mathcal{Q}_{61}^{\circ}$ and $\mathcal{Q}_{91}^{\circ}$ described in [6] are the first five strong generators in the circle.

\section{Notation, definitions and examples}

We denote by $d(\mathbf{p}, \mathbf{q})$ the distance from $\mathbf{p}$ to $\mathbf{q}$, by $S(\mathbf{p}, r)$ the $\operatorname{disk}\{\mathbf{m}: d(\mathbf{p}, \mathbf{m}) \leq r\}$ and by $C(\mathbf{p}, r)$ the circle $\{\mathbf{m}: d(\mathbf{p}, \mathbf{m})=r\}$. A set of $n$ non-overlapping congruent disks all contained in $K$ is called a packing of $K$. For simplicity, we consider in the following that $K$ is either a disk or a polygon whose sides are tangent to a circle. In these cases, $K$ is an erosion-similar body - see [8] - and then the problem of finding the densest packing of $n$ disks in $K$ is equivalent to the maximum separation problem : How to spread $n$ points inside $K$ so that the minimum distance is as large as possible. For this spreading version, an optimal packing is a configuration $\mathcal{P}=\left(\mathbf{p}_{1}, \ldots, \mathbf{p}_{n}\right) \in K^{n}$ which maximizes the function $f(\mathcal{P})=\min _{i \neq j} d\left(\mathbf{p}_{i}, \mathbf{p}_{j}\right)$. Let $g(\mathcal{P})=\min _{i} d\left(\mathbf{p}_{i}, \partial K\right)$ the minimum distance between the points of $\mathcal{P}$ and the boundary of $K$. The function

$$
\omega(\mathcal{P})=\min \left\{\frac{f(\mathcal{P})}{2}, g(\mathcal{P})\right\}
$$



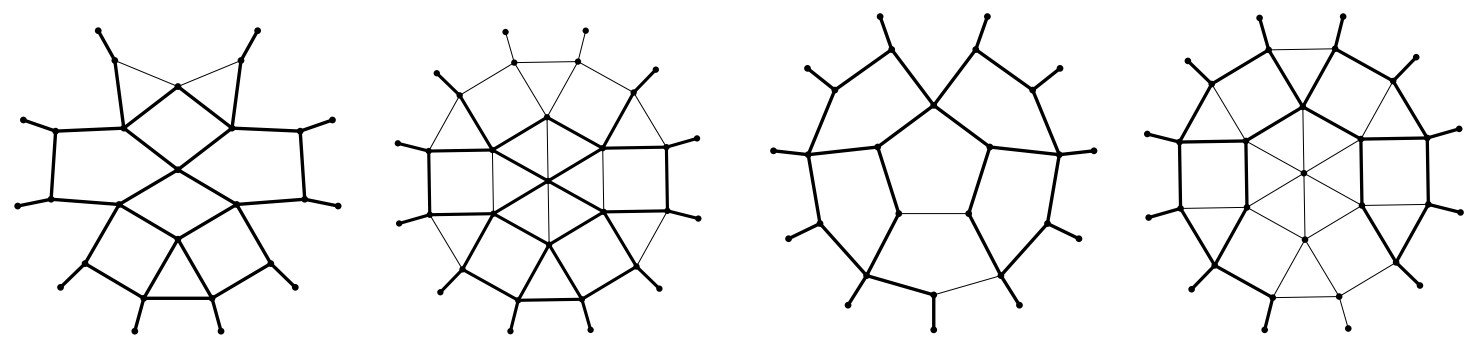

$$
k=17
$$
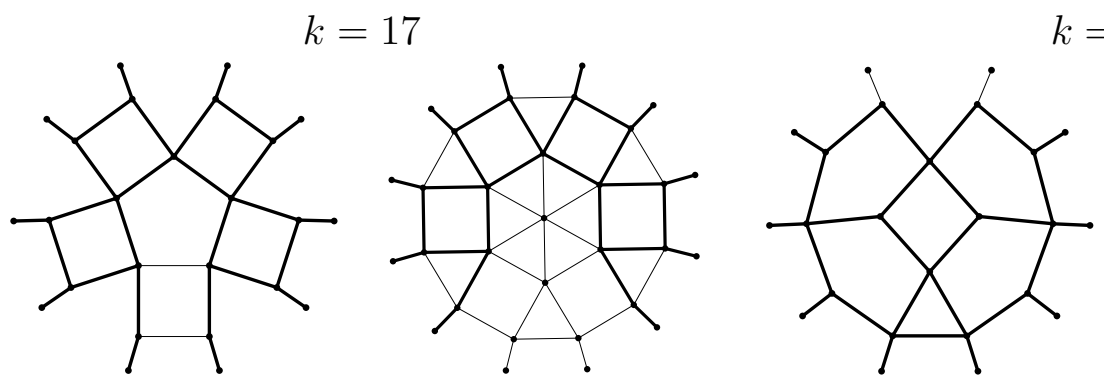

$k=16$
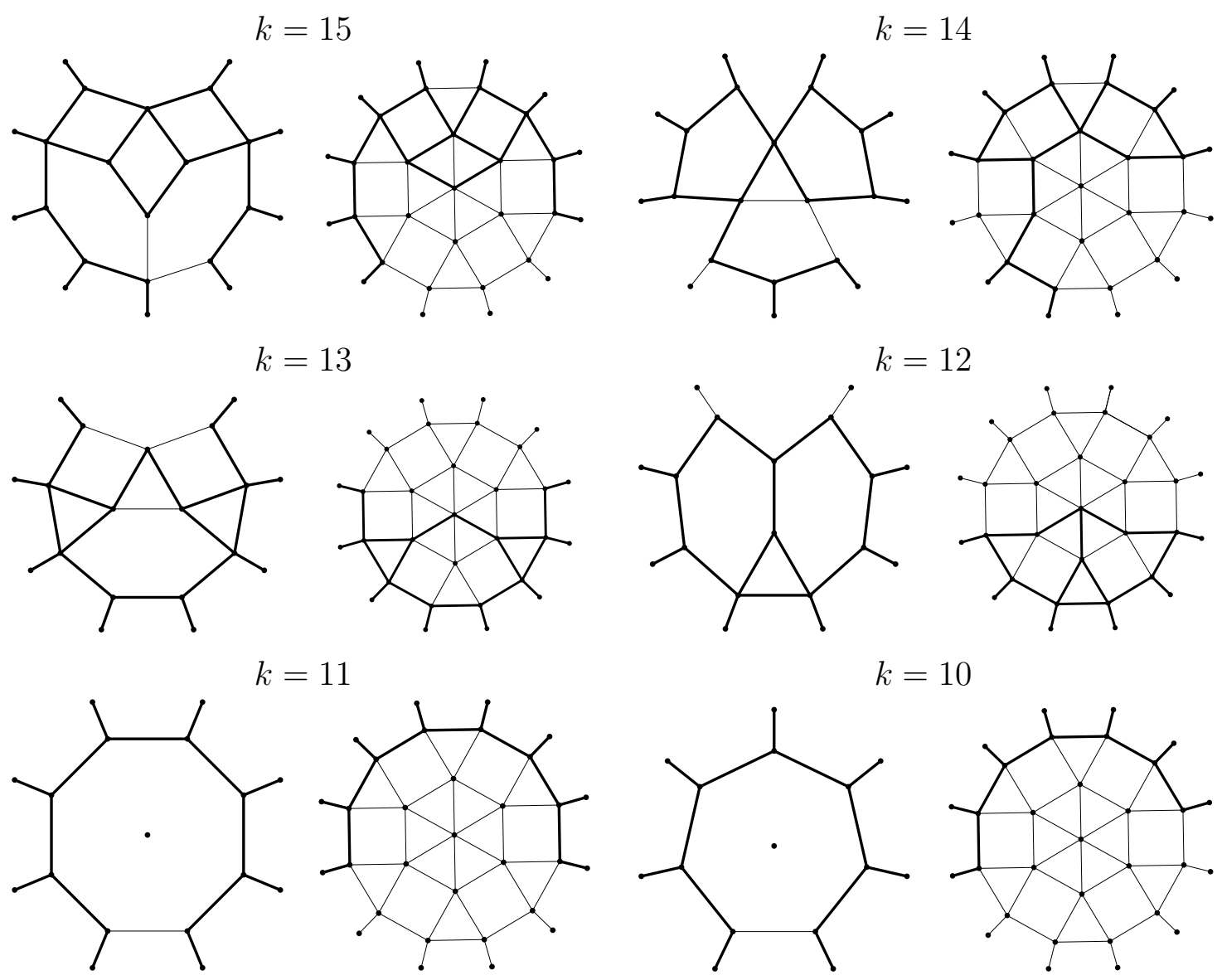

$$
k=9
$$

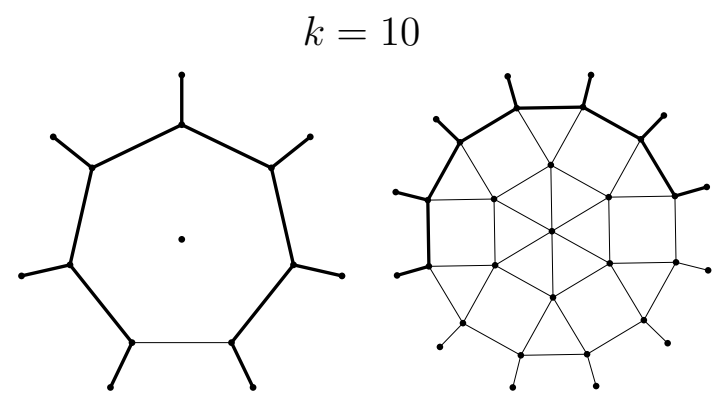

$$
k=8
$$

Figure 2: Largest common subgraphs of the contact graphs of $\mathcal{Q}_{k}^{\circ}$ and $\mathcal{Q}_{19}^{\circ}$ for $17 \geq k \geq 8$. 
is the maximal radius $r$ of the $n$ congruent disks $S\left(\mathbf{p}_{i}, r\right) \subset K$ such that they do not overlap. We often identify the configuration $\mathcal{P}=\left(\mathbf{p}_{1}, \ldots, \mathbf{p}_{n}\right) \in K^{n}$ with the packing of the $n$ disks $S\left(\mathbf{p}_{i}, \omega(\mathcal{P})\right)$. Then an optimal packing $\mathcal{Q}_{n}^{K}$ of $n$ disks in $K$ is a configuration $\mathcal{P}$ of $n$ points in $K$ which maximizes $\omega(\mathcal{P})$.

If $K$ is a polygon, we choose a numbering of its sides and denote them by $K_{1}, \ldots, K_{l}$; if $K$ is a disk, we set $K_{1}=\partial K$. Let us recall that the Danzer graph $\operatorname{Danzer}(\mathcal{P})$ of a packing $\mathcal{P}=\left(\mathbf{p}_{1}, \ldots, \mathbf{p}_{n}\right)$ is obtained by connecting two vertices $\mathbf{p}_{i}$ and $\mathbf{p}_{j}$ when the two disks centered at these points contact each other. An isolated vertex of the Danzer graph is called rattler. The Danzer graph is a subgraph of the contact graph that we now define:

Definition 1 The contact graph $\mathcal{G}=\mathcal{G}(\mathcal{P})=(V, E)$ of a packing $\mathcal{P}=\left(\mathbf{p}_{1}, \ldots, \mathbf{p}_{n}\right)$ in $K$ is the simple, undirected and labelled graph defined by:

- $V=\left\{\mathbf{p}_{1}, \ldots, \mathbf{p}_{n}\right\} \cup\left\{\mathbf{p}_{i}^{j}\right\}$ where $\mathbf{p}_{i}^{j} \in K_{j}$ is the point of contact-provided it exists of the disk $S\left(\mathbf{p}_{i}, \omega(\mathcal{P})\right)$ with the side $K_{j}$ of $K$.

- $E=\left\{\mathbf{p}_{i} \mathbf{p}_{j}: d\left(\mathbf{p}_{i}, \mathbf{p}_{j}\right)=2 \omega(\mathcal{P})\right\} \cup\left\{\mathbf{p}_{i} \mathbf{p}_{i}^{j}\right\}$.

- $\operatorname{label}\left(\mathbf{p}_{i}\right)=0$ and label $\left(\mathbf{p}_{i}^{j}\right)=j$.

Note that the label of the contact graph is not a one-to-one mapping. A Danzer graph is trivially labelled by $\operatorname{label}\left(\mathbf{p}_{i}\right)=0$ when it is considered as a subgraph of the contact graph.

We will denote respectively by $\mathcal{Q}_{n}^{\square}, \mathcal{Q}_{n}^{\triangle}, \mathcal{Q}_{n}^{\circ}$ (one of) the optimal packing(s) of $n$ disks in the unit square, the equilateral triangle, the unit disk; we also denote by $\mathcal{G}_{n}^{\square}, \mathcal{G}_{n}^{\triangle}, \mathcal{G}_{n}^{\circ}$ their respective contact graphs and by $r_{n}^{\square}, r_{n}^{\triangle}, r_{n}^{\circ}$ the disk radii of $\mathcal{Q}_{n}^{\square}, \mathcal{Q}_{n}^{\triangle}, \mathcal{Q}_{n}^{\circ}$. When we consider the packings $\mathcal{Q}_{n}^{\square}, \mathcal{Q}_{n}^{\triangle}, \mathcal{Q}_{n}^{\circ}$ are solutions of the maximal separation problem, we denote by $d_{n}^{\square}, d_{n}^{\triangle}$ and $d_{n}^{\circ}$ the minimum distance between the points of the configurations.

We now recall the definition of an isomorphism between two labelled graphs and the definition of the largest maximal common subgraph of two graphs, see [16].

Definition 2 (a) Two labelled graphs $\mathcal{G}_{1}=\left(V_{1}, E_{1}\right)$ and $\mathcal{G}_{2}=\left(V_{2}, E_{2}\right)$ are said to be isomorphic if there exists a one-to-one mapping $f: V_{1} \longrightarrow V_{2}$ which preserves the adjacency and the labels:

$$
\forall \mathbf{u}, \mathbf{v} \in V_{1},\left(\mathbf{u v} \in E_{1} \Longleftrightarrow f(\mathbf{u}) f(\mathbf{v}) \in E_{2}\right) \text { and label }(f(\mathbf{u}))=\operatorname{label}(\mathbf{u})
$$

(b) We call common subgraph of two graphs $\mathcal{G}_{1}$ and $\mathcal{G}_{2}$ a structure $\left(S_{1}, S_{2}\right)$, where $S_{1}$ is a subgraph of $\mathcal{G}_{1}$ isomorphic to a subgraph $S_{2}$ of $\mathcal{G}_{2}$. A common subgraph is maximal if there is no common subgraph $\left(S_{1}^{\prime}, S_{2}^{\prime}\right)$ of $\mathcal{G}_{1}$ and $\mathcal{G}_{2}$ such that $S_{1}$ is a proper subgraph of $S_{1}^{\prime}$ and $S_{2}$ is a proper subgraph of $S_{2}^{\prime}$. We say that a graph is isomorphic to a common subgraph $\left(S_{1}, S_{2}\right)$ if it is isomorphic to $S_{1}$ (and $\left.S_{2}\right)$. We consider that the size of a graph is the number of its edges and we denote by $\mathcal{G}_{1} \cap \mathcal{G}_{2}$ the largest maximal common subgraph of $\mathcal{G}_{1}$ and $\mathcal{G}_{2}$. There is not always uniqueness of the largest maximal common subgraph (that we shorten by largest common subgraph or l.c.s.). 
We now formalize the definition of weak and strong generations sketched out in the introduction. The points (a) and (b) ensure that, after removing $m-n$ disks of the packing $\mathcal{Q}_{m}$, the $n$ remaining disks move and grow continuously until they become the packing $\mathcal{P}_{n}$. The point (c) gives that during the transformation, the size of the contact graph $\mathcal{G}(t)$ is maximal.

Definition 3 Let $\mathcal{Q}_{m}=\left(\mathbf{q}_{1}, \ldots, \mathbf{q}_{m}\right), \mathcal{P}_{n}=\left(\mathbf{p}_{1}, \ldots, \mathbf{p}_{n}\right)$ be two packings in $K$ with $m \geq n$, and $\mathcal{G}_{1}, \mathcal{G}_{2}$ their respective contact graphs. Let $\mathcal{G}_{1} \cap \mathcal{G}_{2}$ (one of) the l.c.s. of $\mathcal{G}_{1}$ and $\mathcal{G}_{2}$. We say that the packing $\mathcal{Q}_{m}$ generates strongly the packing $\mathcal{P}_{n}$ if, up to a permutation of the points of $\mathcal{Q}_{m}$, there exists a continuous map

$$
\mathcal{Q}: t \in[0,1] \rightarrow \mathcal{Q}(t)=\left(\mathbf{q}_{1}(t), \mathbf{q}_{2}(t), \ldots \mathbf{q}_{n}(t)\right) \in K^{n}
$$

such that

(a) $\mathcal{Q}(0)=\left(\mathbf{q}_{1}, \ldots, \mathbf{q}_{n}\right)$ and $\mathcal{Q}(1)=\left(\mathbf{p}_{1}, \ldots, \mathbf{p}_{n}\right)=\mathcal{P}_{n}$,

(b) $\omega \circ \mathcal{Q}$ is an increasing map,

(c) for each $t \in] 0,1[$, the contact graph $\mathcal{G}(t)$ of the packing $\mathcal{Q}(t)$ is isomorphic to the largest common subgraph $\mathcal{G}_{1} \cap \mathcal{G}_{2}$.

In that case, we note $\mathcal{Q}_{m} \hookrightarrow \mathcal{P}_{n}$.

If (c) is not verified, we say that $\mathcal{Q}_{m}$ generates weakly $\mathcal{P}_{n}$ and we note $\mathcal{Q}_{m} \rightarrow \mathcal{P}_{n}$. An optimal packing $\mathcal{Q}_{m}^{K}$ which generates strongly (resp. weakly) all the optimal packings $\mathcal{Q}_{1}^{K}, \mathcal{Q}_{2}^{K}, \ldots, \mathcal{Q}_{m-1}^{K}$ is called a strong (resp. weak) generator.

When $\mathcal{Q}_{m}$ generates strongly $\mathcal{P}_{n}$, the edges of $\mathcal{G}_{1}$ which are not in the l.c.s. $\mathcal{G}_{1} \cap \mathcal{G}_{2}$ correspond to the contacts which disappear when the transformation begin, either because two disks move away each from the other or because a disk has been removed. The edges of $\mathcal{G}_{2}$ which are not in $\mathcal{G}_{1} \cap \mathcal{G}_{2}$ give the contacts which appear at the end of the transformation, i.e. at $t=1$. For instance, we have illustrated in Fig. 3 a strong generation of $\mathcal{Q}_{11}^{\square}$ from $\mathcal{Q}_{12}^{\square}$; nine edges disappear from the contact graph $\mathcal{G}_{12}^{\square}$ and four edges appear in $\mathcal{G}(t)$ when $t=1$. It is also of interest to recall that if $\mathbf{q}_{i} \mathbf{q}_{j}$ (resp. $\mathbf{q}_{i} \mathbf{q}_{i}^{j}$ ) is an edge of $\mathcal{G}_{1} \cap \mathcal{G}_{2}$, then for each $t \in[0,1]$, the disk of $\mathcal{Q}(t)$ centered at $\mathbf{q}_{i}(t)$ contacts the one centered at $\mathbf{q}_{j}(t)$ (resp. the side $K_{j}$ of the boundary).
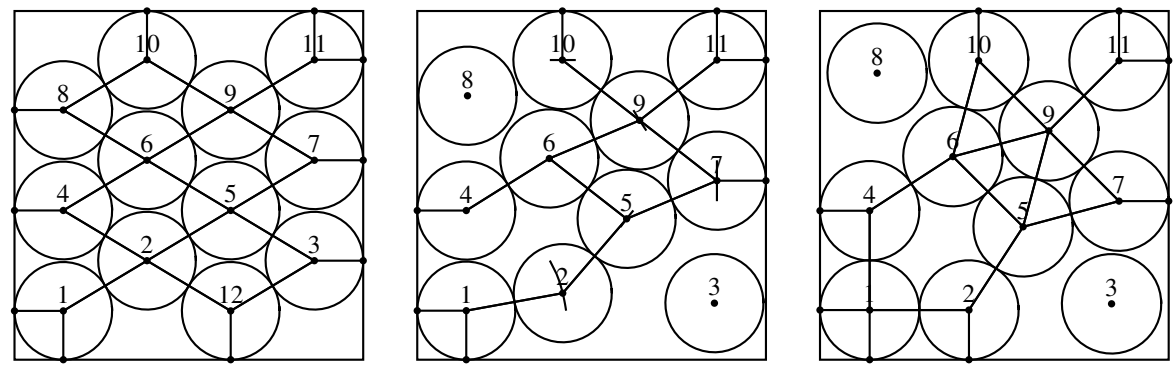

Figure 3: A strong generation $\mathcal{Q}_{12}^{\square} \hookrightarrow \mathcal{Q}_{11}^{\square}$. 
Example 1 We have already seen in the introduction that the optimal packing $\mathcal{Q}_{9}^{\square}$ generates strongly $\mathcal{Q}_{8}^{\square}, \mathcal{Q}_{7}^{\square}$ and $\mathcal{Q}_{6}^{\square}$. It is easy to verify that $\mathcal{Q}_{9}^{\square}$ is a strong generator in the square since it also generates strongly the first optimal packings $\mathcal{Q}_{1}^{\square}, \ldots, \mathcal{Q}_{5}^{\square}$ (displayed in Fig. 8).

Example 2 Here, the domain $K=\Delta$ is the equilateral triangle. We establish the strong generation of the packing $\mathcal{Q}_{13}^{\triangle}=\left(\mathbf{p}_{1}, \ldots, \mathbf{p}_{13}\right)$ from $\mathcal{Q}_{15}^{\triangle}=\left(\mathbf{q}_{1}, \ldots, \mathbf{q}_{15}\right)$ by doing a straight edge and compass construction of the packing $\mathcal{Q}(t)$. In Fig. 4, we have represented in bold lines one of the l.c.s. $\mathcal{G}_{13}^{\triangle} \cap \mathcal{G}_{15}^{\triangle}$. We adopt the numbering of this figure but we consider here that the packing $\mathcal{Q}_{15}^{\triangle}$ is solution of the maximal separation problem in $\Delta$ (the points $\mathbf{q}_{1}, \mathbf{q}_{7}$ and $\mathbf{q}_{10}$ become the three corners of $\Delta$ ). First, we remove $\mathbf{q}_{14}$ and $\mathbf{q}_{15}$. Second, we choose a degree of opening of the compass and use this angle for applying on $\mathbf{q}_{1}, \mathbf{q}_{2}, \ldots, \mathbf{q}_{6}$ an homothety centered at $\mathbf{q}_{1}$ of ratio $u=(1-t) \cdot 1+t \cdot d_{13}^{\triangle} / d_{15}^{\triangle} \geq 1$, we get $\mathbf{q}_{1}(t), \mathbf{q}_{2}(t), \ldots, \mathbf{q}_{6}(t)$. We set $\mathbf{q}_{7}(t)=\mathbf{q}_{7}, \mathbf{q}_{10}(t)=\mathbf{q}_{10}$ and $u^{\prime}=u d_{15}^{\triangle}$ aiming at $u^{\prime}=d_{13}^{\triangle}$ when $t=1$. We find $\mathbf{q}_{8}(t)$ as the point of $C\left(\mathbf{q}_{4}(t), u^{\prime}\right) \cap C\left(\mathbf{q}_{7}(t), u^{\prime}\right)$ which belongs to $\Delta$ and similarly, $\mathbf{q}_{11}(t)=C\left(\mathbf{q}_{6}(t), u^{\prime}\right) \cap C\left(\mathbf{q}_{10}(t), u^{\prime}\right) \cap \Delta$. The intersection of the circle $C\left(\mathbf{q}_{8}(t), u^{\prime}\right)$ with the side $K_{1}=\left[\mathbf{q}_{7}, \mathbf{q}_{10}\right] \subset \partial K$ gives $\mathbf{q}_{9}(t)$ and the intersection of $C\left(\mathbf{q}_{11}(t)\right.$, $\left.u^{\prime}\right)$ with $K_{1}$ gives $\mathbf{q}_{12}(t)$. Finally, we get the point $\mathbf{q}_{13}(t)=C\left(\mathbf{q}_{9}(t), u^{\prime}\right) \cap C\left(\mathbf{q}_{12}(t), u^{\prime}\right) \cap \Delta$. The three points of Definition 3 are verified by the map $t \rightarrow \mathcal{Q}(t)$ and then $\mathcal{Q}_{15}^{\triangleleft} \hookrightarrow \mathcal{Q}_{13}^{\triangle}$.
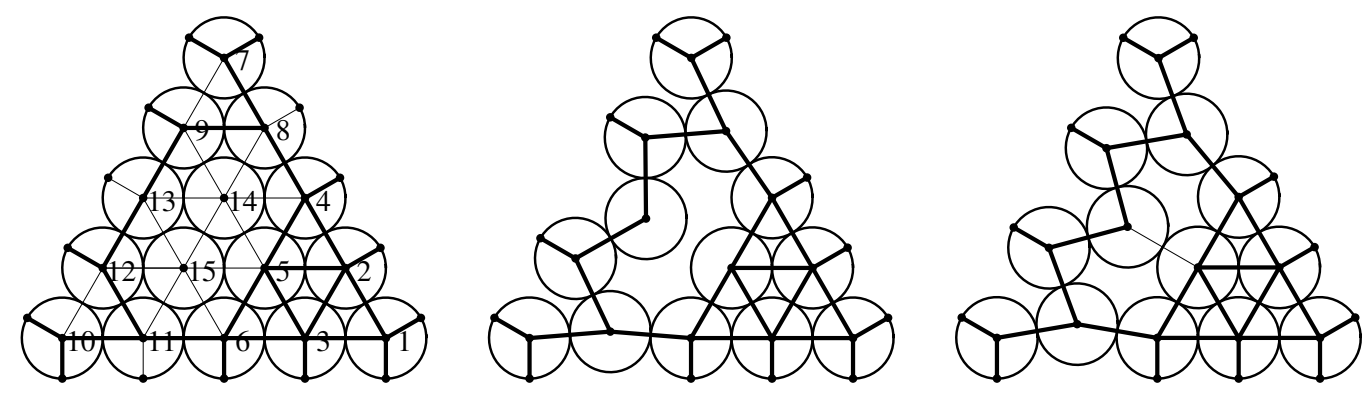

Figure 4: A strong generation of $\mathcal{Q}_{13}^{\triangle}$ from $\mathcal{Q}_{15}^{\triangle}$.

Example 3 The l.c.s $\mathcal{G}_{31}^{\circ} \cap \mathcal{G}_{37}^{\circ}$ displayed in Fig. 5, indicates clearly how $\mathcal{Q}_{37}^{\circ}$ generates strongly $\mathcal{Q}_{31}^{\circ}$. We apply an homothety centered at $O$ on the six vertices of the central hexagon of $\mathcal{G}_{37}^{\circ}$ and we obtain $\mathbf{q}_{k}(t)=2 u r_{37}^{\circ}(\cos k \pi / 3, \sin k \pi / 3)$ where $k \in\{1, \ldots, 6\}$ and $u=(1-t) \cdot 1+t \cdot r_{31}^{\circ} / r_{37}^{\circ}$. Setting $u^{\prime}=2 u r_{37}^{\circ}$, we remark that the vertical straight lines through $\mathbf{q}_{1}(t)$ and $\mathbf{q}_{2}(t)$, i.e. $x= \pm u^{\prime} \cos \pi / 3= \pm u r_{37}^{\circ}$, intersect the circle $C\left(O, 1-u^{\prime} / 2\right)$ at two points $\mathbf{q}_{7}(t)=\left(u r_{37}^{\circ}, y_{7}\right)$ and $\mathbf{q}_{8}(t)=\left(-u r_{37}^{\circ}, y_{8}\right)$ with $y_{7}=y_{8}>0$. We choose $\mathbf{q}_{9}(t)$ in $C\left(\mathbf{q}_{1}(t), u^{\prime}\right) \cap C\left(\mathbf{q}_{7}(t), u^{\prime}\right)$ and accordingly $\mathbf{q}_{10}(t)$ in $C\left(\mathbf{q}_{2}(t), u^{\prime}\right) \cap C\left(\mathbf{q}_{8}(t), u^{\prime}\right)$. Finally, rotations centered at $O=\mathbf{q}_{31}(t)$ of angles $j \pi / 3$ give the other points $\mathbf{q}_{k}(t)$ for $11 \leq k \leq 30$. 

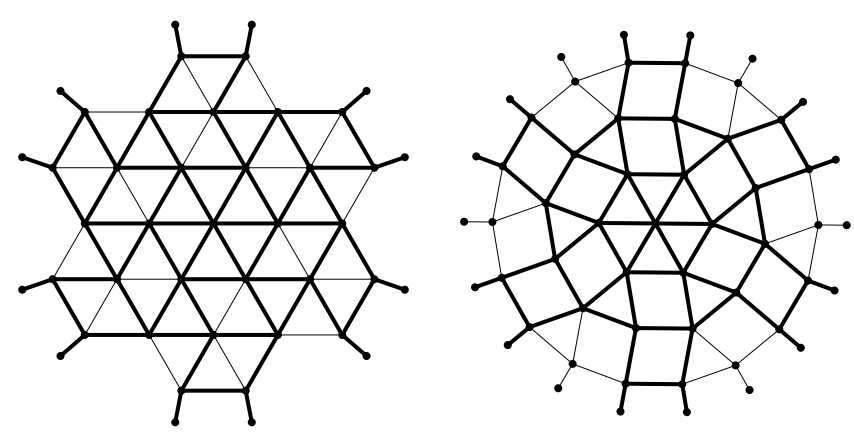

Figure 5: The l.c.s. $\mathcal{G}_{31}^{\circ} \cap \mathcal{G}_{37}^{\circ}$.

\section{Generation in the equilateral triangle}

A list of dense or optimal packings in the equilateral triangle up to $n=21$ disks can be found in [8]. Using their billiard algorithm, Graham and Lubachevsky [4] produced conjectures up to $n=34$ and beyond. In the equilateral triangle, the hexagonal arrangements of $\Delta(k)=k(k+1) / 2$ disks are all optimal - see $[8,11]$ - and we conjecture below that these hexagonal packings are strong generators. For each $n$, we denote by $m_{n}$ the first triangular integer greater than $n$. We notice that up to $n=34$, the Danzer graph of $\mathcal{Q}_{n}^{\triangle}$ is embedded in the Danzer graph of $\mathcal{Q}_{m_{n}}^{\triangle}$-i.e. is isomorphic to a subgraph of this graph and then is embedded in the contact graph $\mathcal{G}_{m_{n}}^{\triangle}$ (all the vertices of the Danzer graph have been labelled with 0). Moreover, it seems to be always possible to find a largest common subgraph $\mathcal{G}_{n}^{\triangle} \cap \mathcal{G}_{m_{n}}^{\triangle}$ which contains the Danzer graph of $\mathcal{Q}_{n}^{\triangle}$. In Fig. 6, we display such l.c.s. for all the non-trivial optimal packings up to $n=20$. Remark that in Fig. 4, we have displayed a l.c.s. $\mathcal{G}_{13}^{\triangle} \cap \mathcal{G}_{15}^{\triangle}$ in which the Danzer graph of $\mathcal{Q}_{13}^{\triangle}$ is not embedded.

Conjecture 2 Each optimal packing $\mathcal{Q}_{n}^{\triangle}$ is strongly generated from the hexagonal packing $\mathcal{Q}_{m_{n}}^{\triangle}$. Moreover, the Danzer graph of $\mathcal{Q}_{n}^{\triangle}$ is embedded in $\mathcal{G}_{m_{n}}^{\triangle}$ and also in one of the largest common subgraphs $\mathcal{G}_{n}^{\triangle} \cap \mathcal{G}_{m_{n}}^{\triangle}$.

Remark 1 When $\mathcal{Q}_{m}^{K}$ generates strongly $\mathcal{Q}_{n}^{K}$, the Danzer graph of $\mathcal{Q}_{n}^{K}$ is not always embedded in the Danzer graph of $\mathcal{Q}_{m}^{K}$, see for instance the two packings $\mathcal{Q}_{15}^{\circ}$ and $\mathcal{Q}_{19}^{\circ}$ in Fig. 2.

Remark 2 In [7], Lubachevsky et al. conjectured that the packings $\mathcal{Q}_{n_{p}(k)}^{\triangle}$ where $n_{p}(k)=$ $\triangle((k+1) p-1)+(2 p+1) \triangle(k))$, have the pattern consisting of one triangle of side $(k+1) p-1$ and $2 p+1$ alternating triangles of sides $k$ with $p-1$ rattlers. These packings are generated from the hexagonal arrangement of $\triangle((p+1) k+p)$ disks after removing a complete row of $(k+1) p$ disks. It is also possible to consider an embedding of the Danzer graph of $\mathcal{Q}_{n_{p}(k)}^{\triangle}$ in the Danzer graph of $\mathcal{Q}_{\triangle((p+1) k+p)}^{\triangle}$ that yields a generation which begin by removing $(k+1) p$ consecutive disks from a side. 

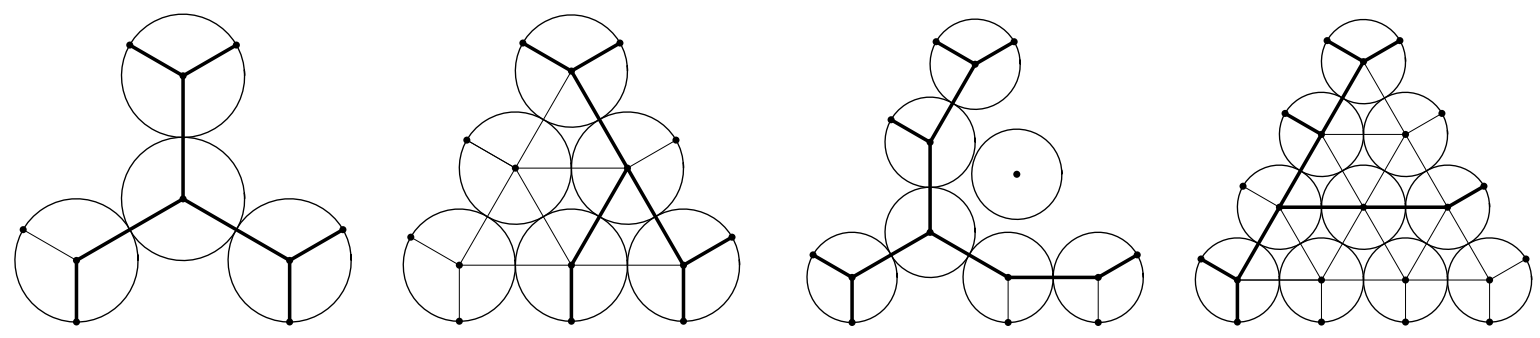

$n=4$
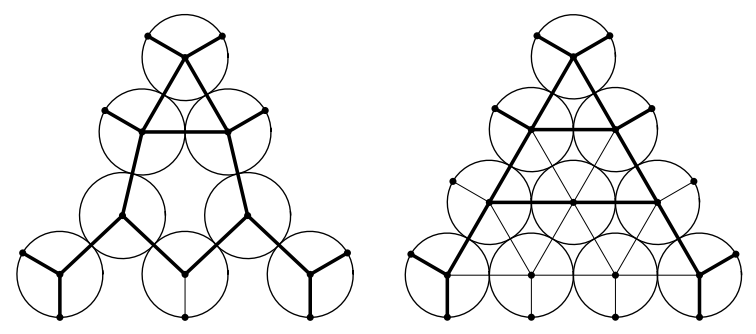

$n=7$

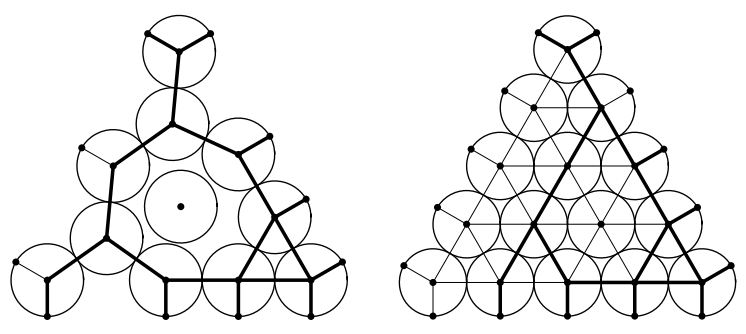

$n=8$

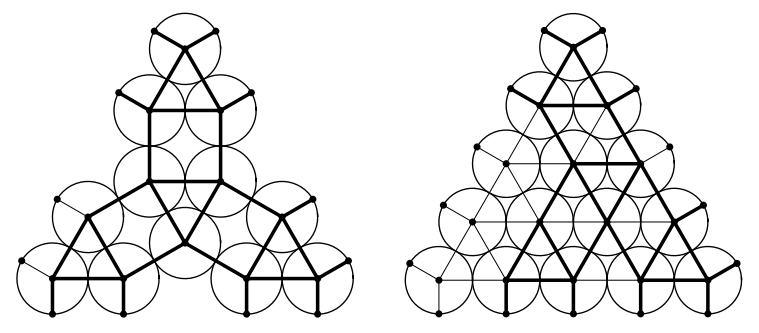

$$
n=11
$$
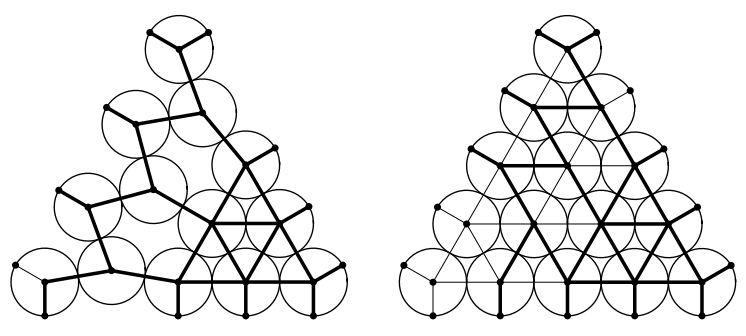

$$
n=12
$$
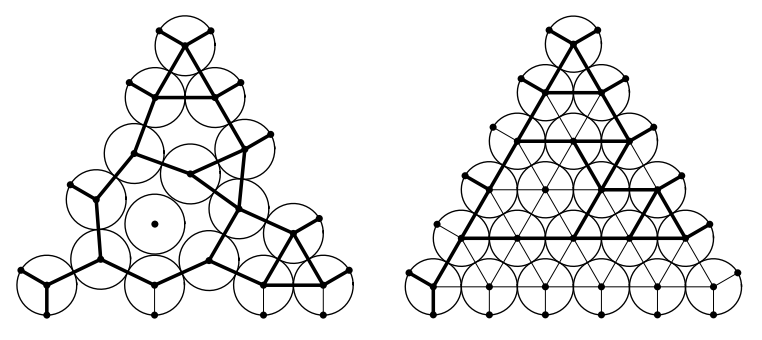

$n=13$

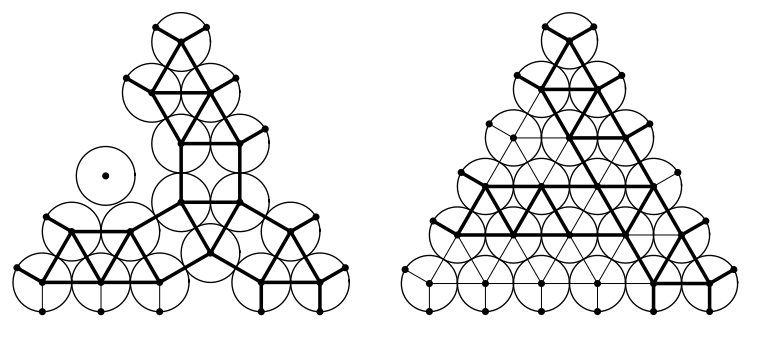

$$
n=16
$$
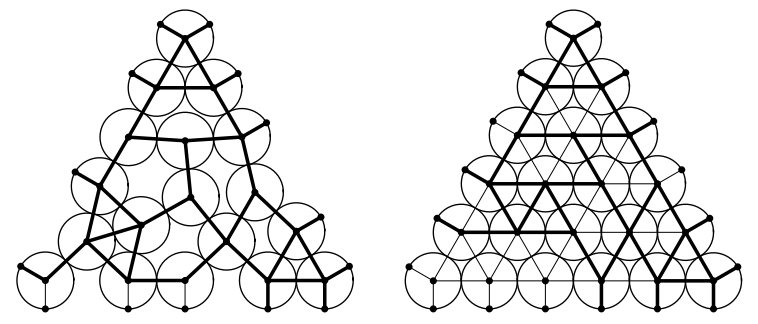

$$
n=18
$$
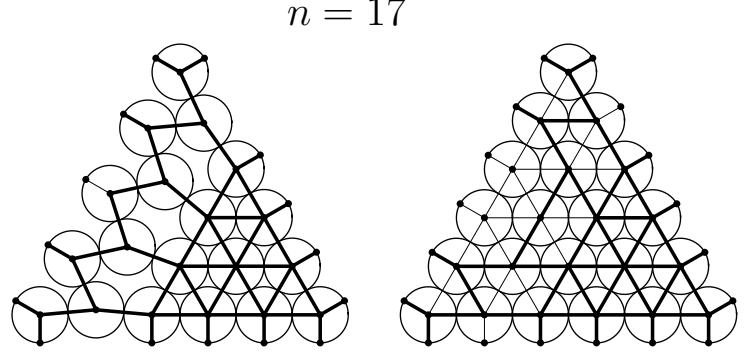

$$
n=19
$$

Figure 6: L.c.s. $\mathcal{G}_{n}^{\triangle} \cap \mathcal{G}_{m_{n}}^{\triangle}$ in which $\operatorname{Danzer}\left(\mathcal{Q}_{n}^{\triangle}\right)$ is embedded. 


\section{Generation in the square}

The problem of packing disks in a square has received a lot of attention, see for instance $[8,15]$. As in the case of the equilateral triangle, the authors of $[5,7,13]$ tried to identify the occurence of repeating patterns. Unfortunately, all these packing patterns cease to be optimal as the numbers of disks exceeds a certain threshold. For instance, the pattern of $n=k(k+1)$ disks which consists of $k+1$ alternating columns with $k$ disks each gives the best known packings up to $k=7$, and non-optimal packings for $k \geq 8$. Nurmela et al. proposed in [10] a pattern $P 1$ by relaxing the ratio $\alpha / \beta$ where $\alpha$ is the number of columns of disks and $\beta$ is the number of rows of disks. See the left side of Fig. 7 where the packing $\mathcal{Q}_{18}^{\square}$ of this pattern is composed of 5 columns and 7 rows of disks. Using the packings of the pattern $P 1$, they get that the maximum number $N_{p}(\sigma)$ of points with mutual distance at least 1 that can be placed into a square of side $\sigma$ verifies

$$
N_{p}(\sigma) \geq \frac{2}{\sqrt{3}}\left(\sigma^{2}+\frac{1-\sqrt{3}}{2} \sigma\right) .
$$

It is more difficult to identify a family of generator in the square. It appears that the optimal packings of the pattern $P 1$ coexist with optimal packings of another pattern that we will denote by $P 2$ : We think that each optimal packing $\mathcal{Q}_{n}^{\square}$ is generated strongly either from an optimal packing of the pattern $P 1$ or from an optimal packing of the pattern $P 2$. Let us define the two patterns, we suppose w.l.o.g. that $b \geq a$.

- $P 1(a, b)$ : We have $a+1$ columns and $b+1$ rows of disks, a disk in a column touches one or two disks in an adjacent column but not other disk in the same column, see for instance $\mathcal{Q}_{12}^{\square}=P 1(3,5)$ in Fig. 3 or $\mathcal{Q}_{18}^{\square}=P 1(4,6)$ in Fig. 7 . A direct calculation shows that the packing $P 1(a, b)$ exists if, and only if, $b \leq \sqrt{3} a$. In this case, the number of disks is $n=\lfloor((a+1)(b+1)+1) / 2\rfloor$ and the disk radius equals $r_{n}=$ $d_{n} /\left(2\left(1+d_{n}\right)\right)$ where $d_{n}=\sqrt{a^{2}+b^{2}} /(a b)$. The first optimal - or presumed optimal packings of the pattern $P 1$ have $n=2,5,6,12,18,27,39,52$ disks, they are obtained respectively for $(a, b)=(1,1),(2,2),(2,3),(3,5),(4,6),(5,8),(6,10),(7,12)$.

- $P 2(a, b)$ : We have also $a+1$ columns and $b+1$ rows of disks, but here $b=2 b^{\prime}+1$ is necessarily odd. The Danzer graph of these packings, as $\mathcal{Q}_{20}^{\square}$ in Fig. 7, forms a pattern of $a b^{\prime}$ diamonds. Now, the existence of the packing $P 2(a, b)$ is equivalent to $b \geq \sqrt{3} a$ and $a>b^{\prime}$. In this case, we have $n=(a+1)\left(b^{\prime}+1\right)$ and $r_{n}=d_{n} /\left(2\left(1+d_{n}\right)\right)$ where $d_{n}=\left(b^{\prime} a-\sqrt{a^{2}-b^{2}+1}\right) /\left(a\left(b^{\prime 2}-1\right)\right)$. We will consider that the square lattice packings of $n=(a+1)^{2}$ disks - which are optimal up to $n=36$, see [9] - belong to the pattern $P 2$. Indeed, except the number of rows of disks, the model is valid when $b^{\prime}=a$ and gives effectively $r_{n}=1 /(2 a+2)$. The first optimal packings - or presumed optimal - of the pattern $P 2$ have $n=4,9,16,20,25,30,36,42$ disks, they are obtained respectively for $(a, b)=(1,3),(2,5),(3,7),(4,7),(4,9),(5,9),(5,11),(6,11)$. 

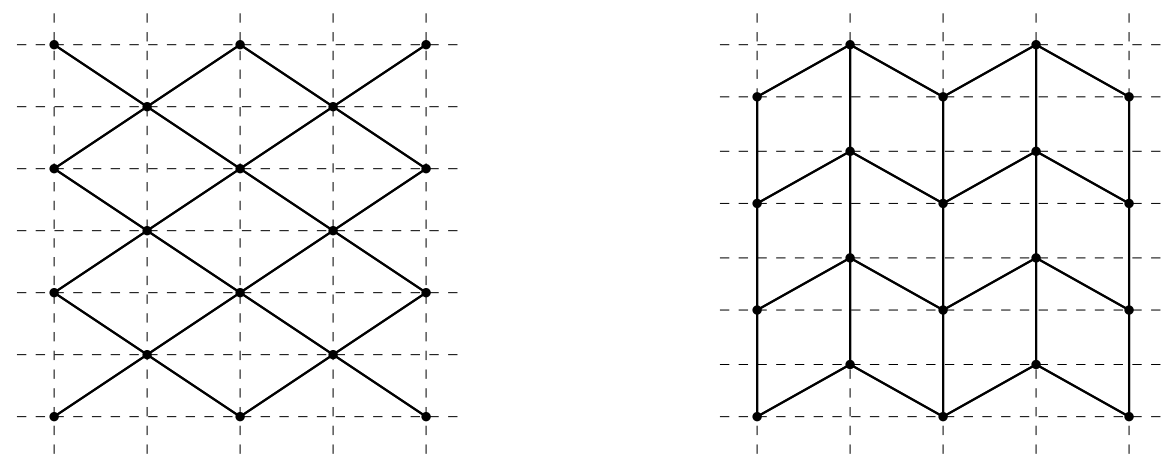

Figure 7: The Danzer graphs of the packings $\mathcal{Q}_{18}^{\square}=P 1(4,6)$ and $\mathcal{Q}_{20}^{\square}=P 2(4,7)$.

Conjecture 3 Each optimal packings $\mathcal{Q}_{n}^{\square}$ is generated strongly either from an optimal packing of the pattern P1 or from an optimal packing of the pattern P2.

We display in Fig. 8-10 all the best known packings up to $n=56$. In each caption, we indicate with $k \hookleftarrow n$ that we think that $\mathcal{Q}_{n}^{\square}$ generates strongly $\mathcal{Q}_{k}^{\square}$. Sometimes, it is possible to generate - weakly or strongly - an optimal packing of the pattern $P 1$ from a packing of the pattern $P 2$ or vice versa. For instance, there is no doubt that $\mathcal{Q}_{20}^{\square}=P 2(4,7)$ generates weakly $\mathcal{Q}_{18}^{\square}=P 1(4,6)$, see Fig. 7 .

In order to maintain Conjecture 1 for the generation in the square, we now describe the infinite subseries of the packing pattern $P 1(a, b)$ given in [10]. Nurmela et al. conjectured that all the packings of this series are optimal and find them by considering the sequence of partial fractions of the continued expansion of $1 / \sqrt{3}$ :

$$
\frac{0}{1}, \frac{1}{1}, \frac{1}{2}, \frac{3}{5}, \frac{4}{7}, \frac{11}{19}, \frac{15}{26}, \frac{41}{71}, \frac{56}{97}, \ldots
$$

In (1), they set $a$ equals to the numerator of each second value starting with $\frac{1}{1}$ and $b$ equals to its denominator and get

$$
(a, b) \in\{(1,1),(3,5),(11,19),(41,71), \ldots\} .
$$

This yields a series of packings $P 1(a, b)$ which begin with $n=2,12,120$, and 1512 points. Szabó [14] proved that the limit density of this packing series is $\pi / \sqrt{12}$. The packing $\mathcal{Q}_{12}^{\square}$ appears to be a strong generator and although it is quite difficult to verify if $\mathcal{Q}_{120}^{\square}$ is a strong generator, we conjecture:

Conjecture 4 The packing series $P 1(a, b)$ obtained by (2) and beginning with $n=2,12$, 120,1512 , is a sequence of strong generators.

Remark 3 In (1), each fourth value starting with 0 gives a fraction $a / b$ with $b$ odd and $b>\sqrt{3} a$, and then a valid packing $P 2(a, b)$. The first three packings of this series have 
1,20 and 2793 points. It is also possible to generate a Farey sequence which converges to $1 / \sqrt{3}$ using the classical method: Let $A=\frac{0}{1}, B=\frac{1}{1}$ and $X=A \oplus B$, where the sum $\oplus$ is defined by $\frac{c}{d} \oplus \frac{e}{f}=\frac{c+e}{d+f}$. If $X<\frac{1}{\sqrt{3}}$ then we set $A:=X$, else $B:=X$. By iterating this process, we find the sequence

$$
\frac{0}{0}, \frac{1}{1}, \frac{1}{2}, \frac{2}{3}, \frac{3}{5}, \frac{4}{7}, \frac{7}{12}, \frac{11}{19}, \frac{15}{26}, \frac{26}{45}, \frac{41}{71}, \frac{56}{97}, \frac{97}{168}, \ldots
$$

This Farey sequence converges slower than (1) but gives more optimal packings : The fractions $\frac{a}{b}$ of (3) which verify $b \leq \sqrt{3} a$ give the best known packings of $P 1$ of $n=$ $2,6,12,52,120,621,1512,8281$ disks. The fractions $\frac{a}{b}$ of (3) which verify $b \geq \sqrt{3} a$ and $b$ odd give the best known packings of $P 2$ of $n=20,2793$ disks. We think that all the valid "Farey packings" are optimal.

We conclude this remark by noticing that the sum $\oplus$ has already been used in [15]: In order to propose conjectural infinite grid packing sequences, the authors consider new subseries of the pattern $P 1(a, b)$. For instance, the sums of two consecutive elements of (2) lead to the packing series $P 1(4,6), P 1(14,24), P 1(52,90), \ldots$ which begin with $n=$ $18,188,2412$ disks.
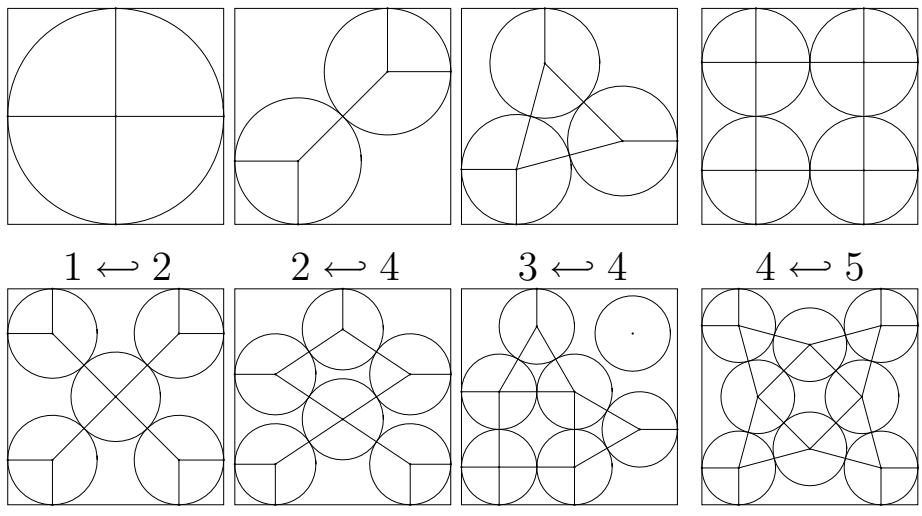

$4 \hookleftarrow 5$
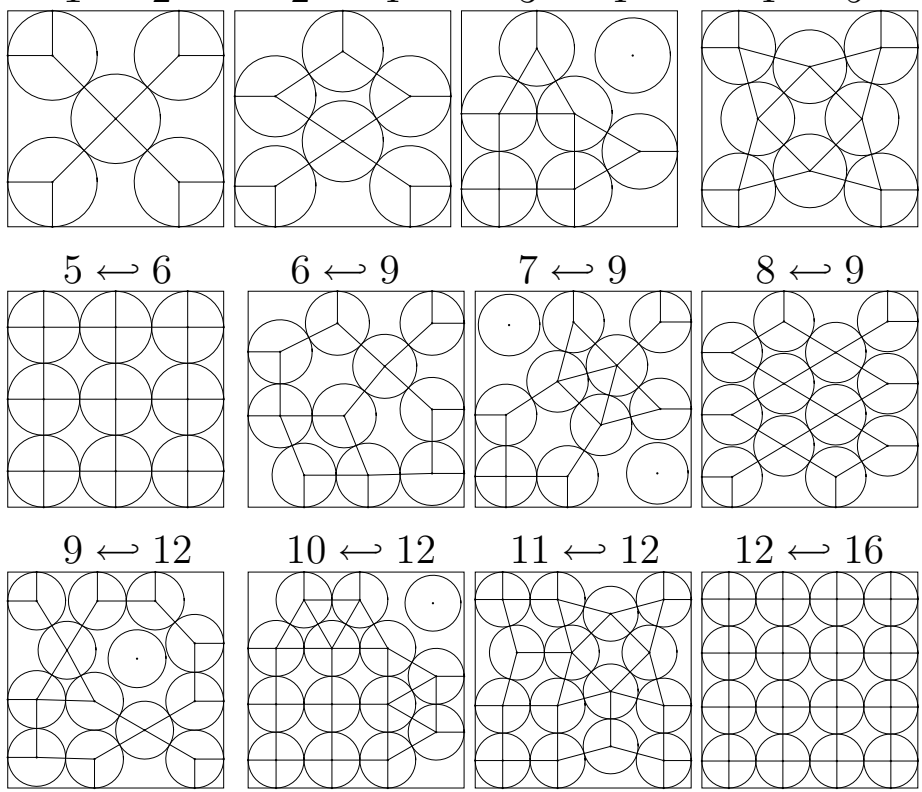

$11 \hookleftarrow 12 \quad 12 \hookleftarrow 16$

$$
13 \hookleftarrow 16 \quad 14 \hookleftarrow 16 \quad 15 \hookleftarrow 16 \quad 16 \hookleftarrow 20
$$

Figure 8: Optimal packings $\mathcal{Q}_{k}^{\square}$ for $k=1$ to 16 . 

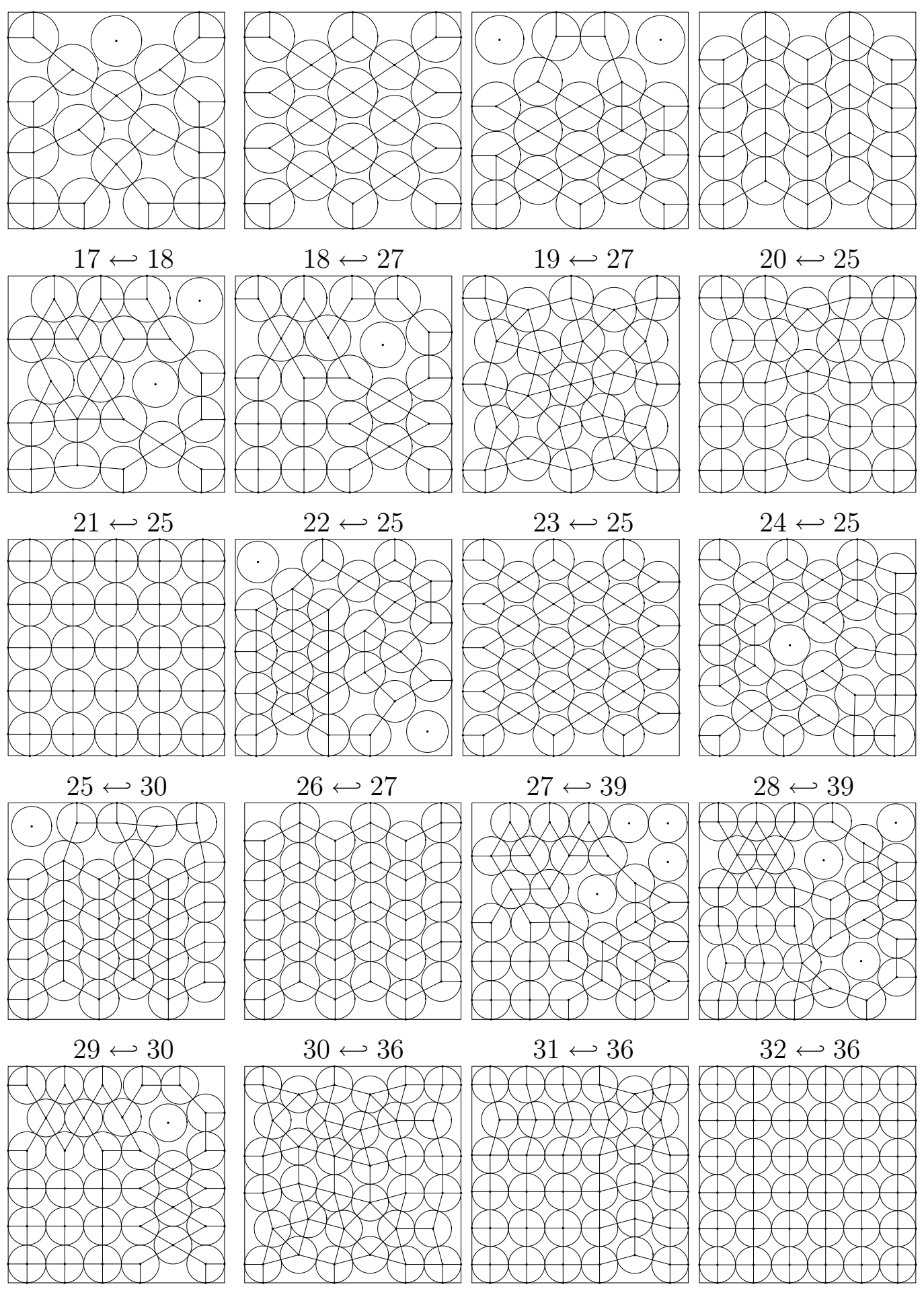

$33 \hookleftarrow 36$

$34 \hookleftarrow 36$

$35 \hookleftarrow 36$

$36 \hookleftarrow 42$

Figure 9: Best known packings $\mathcal{Q}_{k}^{\square}$ for $k=17$ to 36 . 


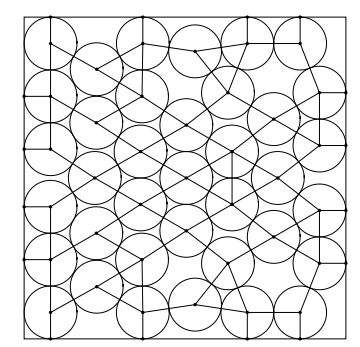

$37 \hookleftarrow 39$

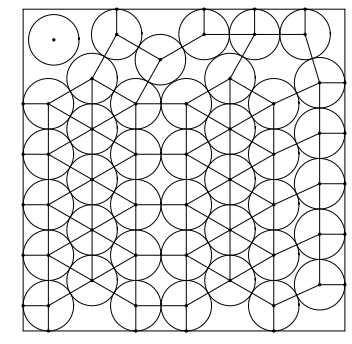

$41 \hookleftarrow 52$
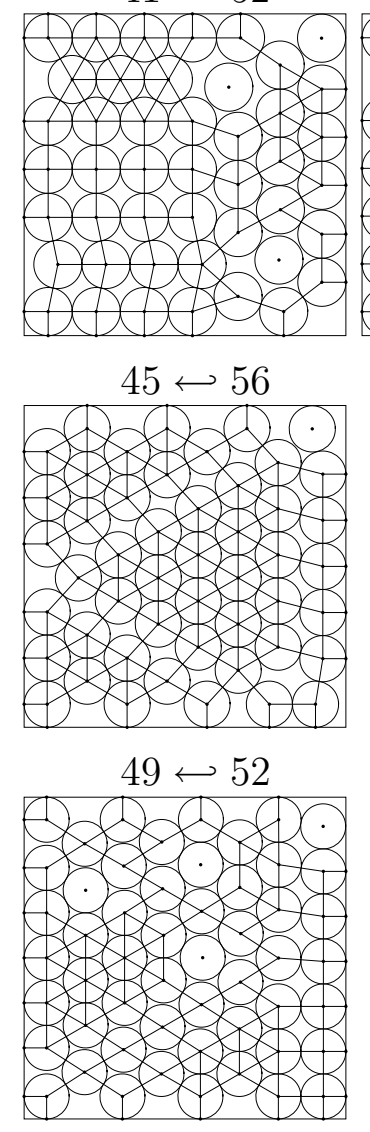

$$
53 \hookleftarrow 99
$$
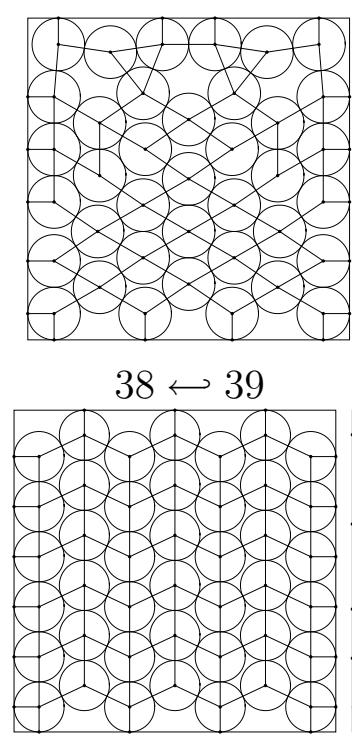

\begin{abstract}
$42 \hookleftarrow 56$
\end{abstract}

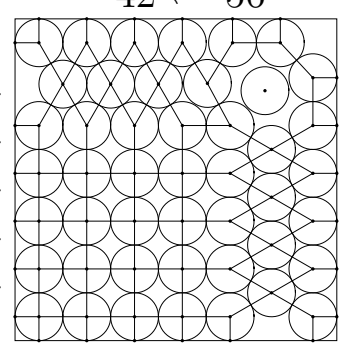

$46 \hookleftarrow 56$
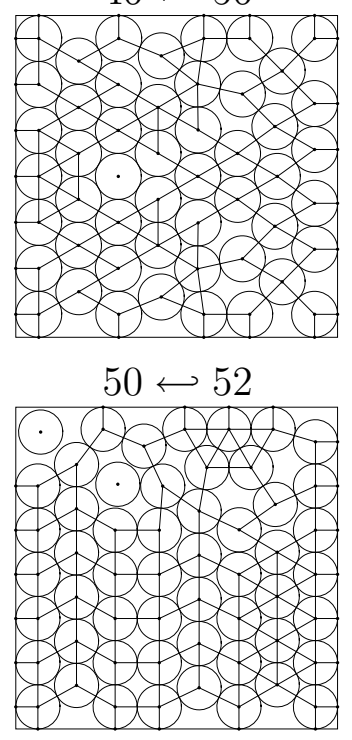

$54 \hookleftarrow 143$

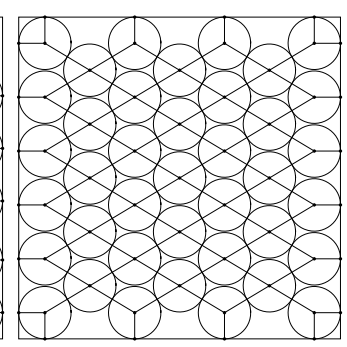

$39 \hookleftarrow 52$

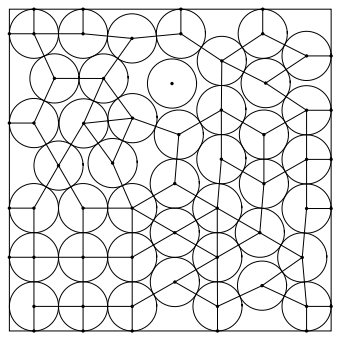

$43 \hookleftarrow 56$

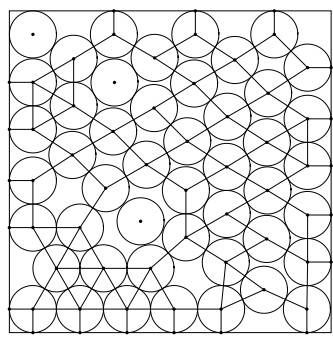

$47 \hookleftarrow 52$

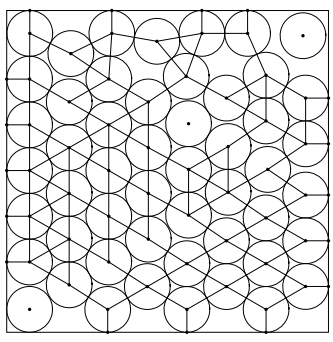

$51 \hookleftarrow 52$

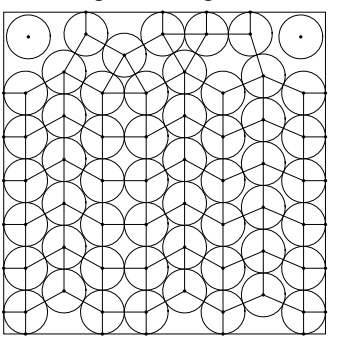

$55 \hookleftarrow 143$

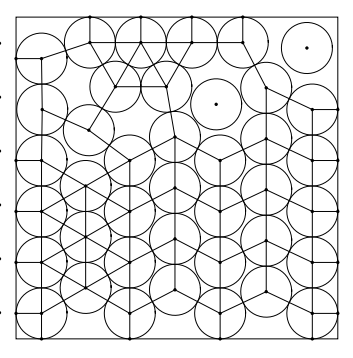

$40 \hookleftarrow 42$

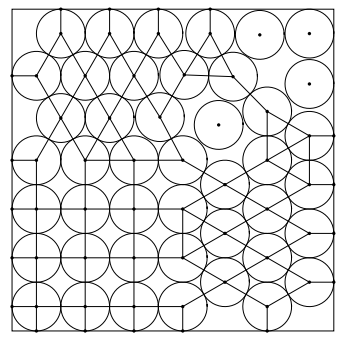

$44 \hookleftarrow 56$

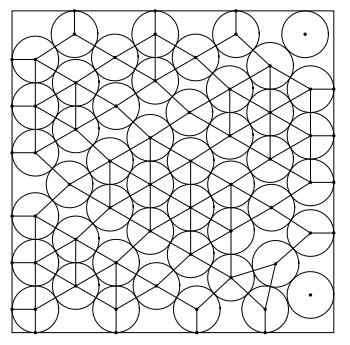

$48 \hookleftarrow 52$
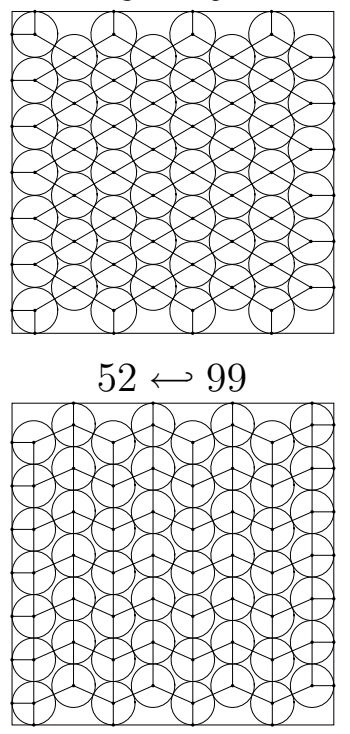

$56 \hookleftarrow 143$

Figure 10: Best known packings $\mathcal{Q}_{k}^{\square}$ for $k=37$ to 56 . 


\section{References}

[1] H.T. Croft, K.J. Falconer and R.K. Guy, Unsolved Problems in Geometry, Springer Verlag, New York, 1991.

[2] E. Friedman, website, http://www.stetson.edu/ efriedma/packing.html.

[3] M. Garey and D. Johnson, Computers and Intractability: a guide to the theory of NP-completeness, Freeman (1979).

[4] R.L. Graham and B.D. Lubachevsky, Dense packings of equal disks in an equilateral triangle: from 22 to 34 and beyond., Electron. J. Combin. 2 (1995), \#A1.

[5] R.L. Graham and B.D. Lubachevsky, Repeated patterns of dense packings of equal disks in a square. Electron. J. Combin. 3 (1996), \#R16.

[6] B.D. Lubachevsky and R.L. Graham, Curved hexagonal packings of equal disks in a circle, Discrete Comput. Geom. 18 (1997), 179-194.

[7] B.D. Lubachevsky, R.L. Graham and F.H. Stillinger, Patterns and structures in disk packings, Period. Math. Hungar. 34 (1997), no. 1-2, 123-142.

[8] H. Melissen, Packing and covering with circles, Ph.D. thesis, Utrecht University, 1997.

[9] K.J. Nurmela and P.R.J. Östergård, More optimal packings of equal circles in a square. Discrete Comput. Geom. 22 (1999), no. 3, 439-457.

[10] K.J. Nurmela, P.R.J. Östergård and R. aus dem Spring, Asymptotic behavior of optimal circle packings in a square, Can. Math. Bull. 42 (1999), 380-385.

[11] N. Oler, A finite packing problem, Can. Math. Bull. 4 (1961), 153-155.

[12] E. Specht, website, http://hydra.nat.uni-magdeburg.de/packing/.

[13] P.G. Szabó, Some new structures for the "equal circles packing in a square" problem, Proceedings of the XXIV Hungarian Operations Research Conference (Veszprém, 1999). CEJOR Cent. Eur. J. Oper. Res. 8 (2000), no. 1, 79-91.

[14] P.G. Szabó, Optimal substructures in optimal and approximate circle packings, Beitrage zur Algebra und Geometrie 46 (2005), no. 1, 103-118.

[15] P.G. Szabó , M.Cs. Markót, T. Csendes, E. Specht, L.G. Casado and I. Garcia, New approaches to circle packing in a square (with program codes), Springer Optimization and Its Applications 6, Springer, New york, 2007.

[16] G. Valiente, Algorithms on trees and graphs, Springer-Verlag, Berlin, 2002. 